\title{
TOWARDS PATHOGEN DETECTION VIA OPTICAL INTERROGATION OF MAGNETIC MICRODISCS
}

\author{
K.Y. Castillo-Torres ${ }^{1 *}$, N. Garraud ${ }^{1}$, E. S. McLamore ${ }^{2}$, and D.P. Arnold ${ }^{l}$ \\ ${ }^{1}$ Interdisciplinary Microsystems Group, Dept. Electrical and Computer Engineering \\ ${ }^{2}$ Dept. Agricultural and Biological Engineering \\ University of Florida, Gainesville, Florida, USA
}

\begin{abstract}
We present progress in the development of a micro/nanobased scheme for portable, rapid, low-cost detection of foodborne bacterial pathogens, such as Escherichia coli, Listeria monocytogenes, or Salmonella. The system uses magnetic microdiscs that are functionalized for selective binding to target pathogens. The presence of a target pathogen is evaluated by optically monitoring the rotation of the microdiscs when subjected to a rotating magnetic field. This article summarizes the following advancements: (1) wafer-level microfabrication of 600 million gold-coated microdiscs per substrate; (2) functionalization of the microdiscs with DNA aptamers and subsequent binding to E. coli $\mathrm{O} 157: \mathrm{H} 7$; and (3) magnetic manipulation of the bound bacteria.
\end{abstract}

\section{INTRODUCTION}

Outbreaks of foodborne pathogens are becoming increasingly frequent and represent a growing threat to public health [1]. The existing approaches for pathogen screening rely on highly skilled personnel, expensive laboratory equipment, and long time frames (e.g. 8-24 hr for DNA amplification and PCR detection) [2].

The proposed system involves optical measurements of the rotational dynamics of suspended magnetic microdiscs functionalized with pathogen-binding aptamers. The magnetic microdiscs $(1.5 \mu \mathrm{m}$ in diameter) have low-aspect-ratio and are fabricated using a soft ferromagnetic material: $70 \mathrm{~nm}$ permalloy $\left(\mathrm{Ni}_{80} \mathrm{Fe}_{20}\right)$ with a $5 \mathrm{~nm}$ surface coating of gold (Au). These microdiscs exhibit a closed magnetic spin arrangement (spin vortex) with zero magnetic stray field. This behavior is critical to avoid disc agglomeration when in free suspension [3] (i.e. fluidic samples). With very high surface area for functionalization and volumes $10,000 x$ larger than commonly used superparamagnetic nanoparticles, these micro-sized microdiscs are well suited for tagging, trapping, actuating, or interrogating bacterial pathogens, which have comparable sizes to bacterial pathogens in the range of $0.3-1 \mu \mathrm{m}$ [4]. These magnetic microdiscs tend to align their in-plane magnetic easy-axis to an external rotating magnetic field, $\overrightarrow{\mathrm{B}_{\text {ext }}}$, hence they can be actuated synchronously to act as an "optical shutter" [5] (Fig. 1).

The experimental setup proposed in this work takes advantage of the synchronous behavior between the rotating microdiscs and the rotating magnetic field ("optical shuttering"). Here, a combination of magnetic actuation and optical detection is performed using two orthogonal magnetic coils, a laser, and a photodiode to control and monitor the light transmission modulation, or light intensity, of the laser (Fig. 1). A phase-lag exists between the rotating magnetic field and the disc orientation, which depends on the magnetic field amplitude, rotation frequency, fluid viscosity, and disc hydrodynamic diameter (Fig. 2).

Previous work has shown how this apparatus is sensitive to its environment and can be used to measure fluid viscosity [5]. Therefore, this approach of monitoring the light transmission modulation will be adapted for pathogen detection applications by characterizing the light intensity sensed by the photodiode for different control and target bacteria concentrations when exposed to the biofunctionalized suspended microdiscs. For pathogenic detection, the goal is to alter the hydrodynamic diameter via specific binding with target bacteria, which will dramatically change the rotational dynamics.

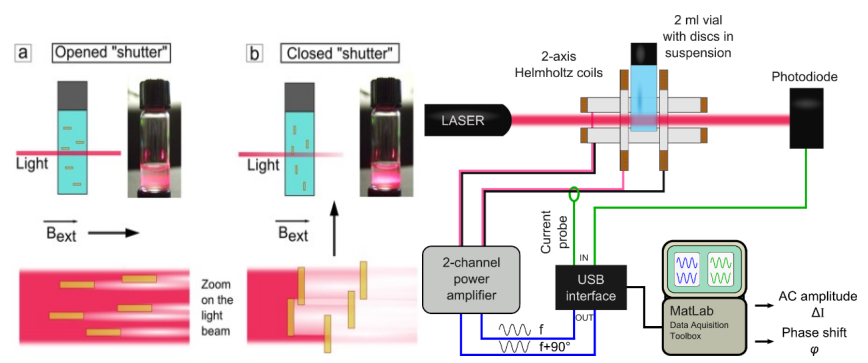

Figure 1: "Optical shutter" behavior of microdiscs actuated by the rotating magnetic field (left) and proposed pathogen detector apparatus (right). (Reproduced from [5] with permission of AIP Publishing)
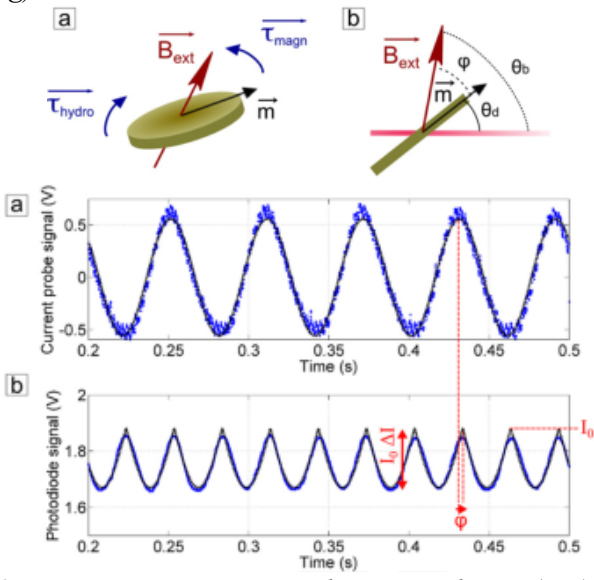

Figure 2: Torques acting on the microdiscs (top) and light intensity measurement example (bottom). (Reproduced from [5] with permission of AIP Publishing)

\section{RESULTS}

\section{Microfabrication process}

Dense arrays of magnetic microdiscs are microfabricated on $100 \mathrm{~mm}$ Si substrates, yielding 600 million microdiscs $(0.86 \mathrm{mg})$ per wafer using standard microfabrication techniques, such as photolithography, thin metal deposition, and lift-off. Here, a hexagonal array of circular polymer pillars $(1.5 \mu \mathrm{m}$ in diameter; $3.0 \mu \mathrm{m}$ minimum center-to-center spacing) is patterned using a positive photoresist in an area of $\sim 20 \mathrm{~cm}^{2}$ of the wafer. In order to obtain higher resolution and re-entrant sidewalls for the photoresist pillars, an image reversal recipe is utilized before sputtering. Next, a metal stack of $\mathrm{Au}(5 \mathrm{~nm}) / \mathrm{Ni}_{80} \mathrm{Fe}_{20}(70 \mathrm{~nm}) / \mathrm{Au}$ $(5 \mathrm{~nm})$ is sputtered. Finally, the magnetic microdiscs are lifted off from the substrate using ultrasonic agitation with acetone and triple-washed in DI water (Fig.3). 

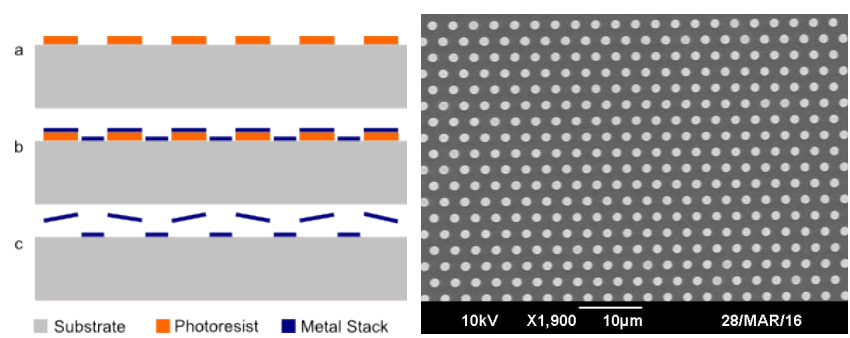

Figure 3: (left) Microfabrication process: (a) photoresist pillars, (b) metal deposition, (c) lift-off; and (right) SEM image of dense hexagonal array of microdiscs on photoresist pillars.

\section{Disc functionalization}

After the disc microfabrication, these are functionalized for selective binding to a biological target (Fig.4). Here, we use Eschericia coli (E. coli) O157:H7, a common food-borne bacterial pathogen, as a demonstration pathogen. The gold surfaces of the microdiscs are functionalized with a DNA aptamer (21 mer), which functions as a capture probe. The aptamer discriminates $E$. coli $\mathrm{O} 157: \mathrm{H} 7$ from other gram-negative bacteria by forming a covalent bond with cell surface $\mathrm{O}$-antigen structures (binding with O-antigen occurs at the 5' end of the aptamer). The DNA contains a thiol group and a six-carbon spacer at the 3' terminal end, which ensures adequate mobility for bacteria binding as demonstrated in [6]. The aptamers are in a dithiotriotol HEPES buffer at $20 \mathrm{nM}$ concentration and $\mathrm{pH}$ of 7.1. Here, $10 \mu \mathrm{L}$ of the DNA aptamer solution is added to an $8 \mathrm{~mL}$ solution of discs (12.5 million discs per $\mathrm{mL}$ ). The aptamers adsorb to the discs via gold-thiol binding.

\section{Bacteria binding and magnetic manipulation}

After functionalization, the microdiscs are exposed to GFPexpressing E. coli O157:H7. The bacteria concentration was only $1 \mathrm{CFU} / \mathrm{mL}$, which is at the lower limit of detectability for other biosensors. Here, $10 \mu \mathrm{L}$ of the bacteria suspension was added to the $8 \mathrm{~mL}$ solution of functionalized discs. In this experiment, the concentration of magnetic microdiscs was much higher than $E$. coli in order to maximize capture of target cells. After $10 \mathrm{~min}$ incubation, the discs were concentrated using an external magnet (Fig. 5), and $1 \mu \mathrm{L}$ was pipetted and then deposited on a coverslip. Figure 6 shows representative images of the E. coli successfully bound to the aptamer-functionalized discs. Here the dark areas are clusters of discs (magnetically agglomerated in this case), and the bright green spots are the GFP E. coli.

\section{OUTLOOK}

With the ability to synthesize large batches of biofunctionalized magnetic microdiscs, ongoing efforts are focused on optimizing the concentration of discs and also detection of bacteria via the optical interrogation of magnetic microdiscs under rotation.

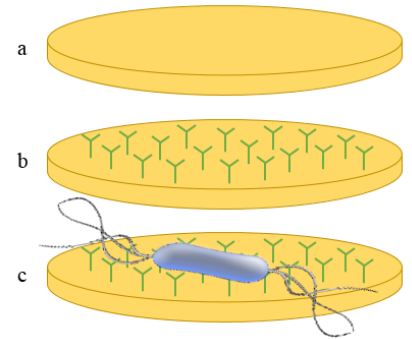

Figure 4: Schematic of binding process: (a) gold coated magnetic microdisc, (b) biofunctionalized microdisc with DNA aptamer capture probe, and (c) bound cell (E. coli).

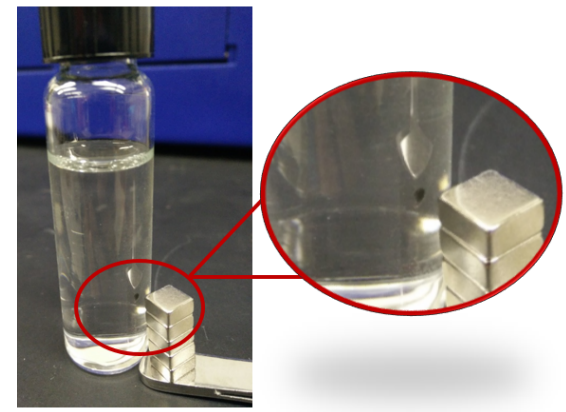

Figure 5: Microdiscs trapping and concentration using permanent magnet before fluorescent observation under microscope.
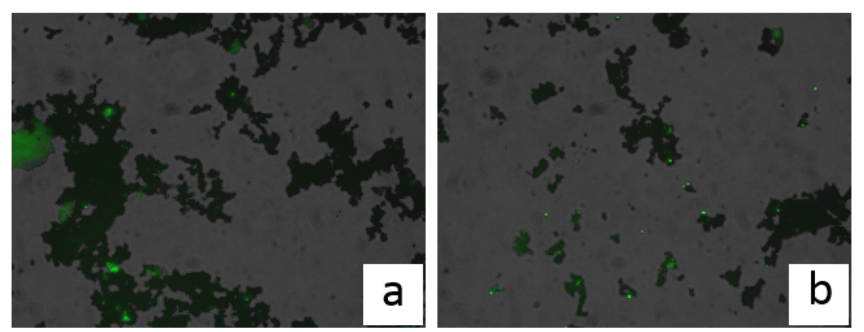

Figure 6: Fluorescent/optical image overlay of magnetically concentrated magnetic microdiscs (dark areas) bound to GFP E. coli O157:H7 (bright fluorescent green spots).

\section{ACKNOWLEDGMENTS}

This work was supported in part by the University of Florida Office of Technology Licensing. The authors thank Prof. Z. Hugh Fan for use of the fluorescent microscope and the staff of the UF Nanoscale Research Facility for assistance in the microfabrication. Travel support has been generously provided by the Transducer Research Foundation.

\section{REFERENCES}

[1] N. Bakalar, "120 Multistate Outbreaks: Tip of Iceberg in Food-borne Infection," Nytimes.com (2015). Available online: http://www.nytimes.com/2015/12/15/science/120multistate-outbreaks-tip-of-iceberg-in-food-borneinfection.html. [Accessed: 15-Mar-2016].

[2] J. Yoon and B. Kim, "Lab-on-a-Chip Pathogen Sensors for Food Safety," Sensors, 12, no. 12, pp. 10713 (2012).

[3] N. Garraud and D. P. Arnold, "Experimental investigation of magnetic anisotropy in spin vortex discs," J. Appl. Phys., 115, 17D128 (2014).

[4] I. Sinn et al., "Asynchronous Magnetic Bead Rotation Microviscometer for Rapid, Sensitive, and Label-Free Studies of Bacterial Growth and Drug Sensitivity," Anal. Chem., 84, pp. 5250 (2012).

[5] N. Garraud and D. P. Arnold, "Characterization of fluids via measurement of the rotational dynamics of suspended magnetic microdiscs," J. Appl. Phys., 117, no. 17, 17B320, (2015).

[6] Y. Wang, F. Zhou, X. Liu et al., "Aptamer-modified micro/nanostructured surfaces: efficient capture of Ramos cells in serum environment," ACS Applied Materials \& Interfaces. 5(9), 3816 (2013).

\section{CONTACT}

*K.Y. Castillo-Torres, keishact@ufl.edu 